\title{
Нелинейно-оптические свойства квантовых точек CdS/ZnS в матрице из высокомолекулярного поливинилпирролидона
}

\author{
(C) А.С. Кулагина ${ }^{1,2}$, С.К. Евстропьев ${ }^{2,3}$, Н.Н. Розанов ${ }^{2,3,4}$, В.В. Власов ${ }^{2}$ \\ ${ }^{1}$ Санкт-Петербургский национальный исследовательский Академический университет \\ Российской академии наук, \\ 194021 Санкт-Петербург, Россия \\ ${ }^{2}$ Санкт-Петербургский национальный исследовательский университет \\ информационных технологий, механики и оптики, \\ 197101 Санкт-Петербург, Россия \\ ${ }^{3} \mathrm{AO}$ „ГОИ им. С.И. Вавилова“, \\ 199053 Санкт-Петербург, Россия \\ ${ }^{4}$ Физико-технический институт им. А.Ф. Иоффе Российской академии наук, \\ 194021 Санкт-Петербург, Россия \\ E-mail: a.s.panfutova@gmail.com
}

(Получена 12 сентября 2017 г. Принята к печати 26 сентября 2017 г.)

Синтезированы и исследованы нелинейные свойства золей, содержащих полупроводниковые квантовые точки $\mathrm{CdS} / \mathrm{ZnS}$ типа ядро/оболочка, представляющие интерес для широкого круга приложений нанофотоники. Получены квантовые точки размером меньшем боровского радиуса экситона, и поэтому в них реализуется выраженный квантово-размерный эффект. Нелинейно-оптические свойства низкоконцентрированных золей исследовались под действием лазерных импульсов с длиной волны излучения 532 нм и длительностью 5 нс по методу $z$-сканирования. Получены зависимости нелинейно-оптических коэффициентов от концентрации квантовых точек CdS/ZnS. Представлена зависимость коэффициентов двухфотонного поглощения от интенсивности, определяющая границу влияния нелинейностей высших порядков на нелинейное пропускание образцов. Обсуждаются механизмы оптического ограничения, проявляющиеся в синтезированных золях: двухфотонное поглощение, нелинейная рефракция, нелинейное рассеяние.

DOI: 10.21883/FTP.2018.08.46211.8724

\section{1. Введение}

Полупроводниковые квантовые точки (КТ), такие как $\mathrm{CdS}$, обладают сильными нелинейными оптическими свойствами как при пикосекундном, так и при наносекундном воздействии [1-10]. Нелинейные структуры, содержащие КТ в золях на основе поливинилпирролидона (PVP) или пленках, перспективны в оптическом ограничении лазерного излучения, а также представляют интерес при разработке твердотельных нелинейных структур [2]. Для достижения высоких нелинейнооптических характеристик ранее использовались композитные материалы с высоким содержанием КT $\mathrm{CdS}-$ см., например, $[1,2,4,7-9]$. В композиционных материалах с высоким содержанием квантовых точек часто протекают процессы роста наночастиц, их агрегации и коалесценции, образования микронеоднородной структуры. Поэтому концентрационные зависимости оптических свойств в концентрированных материалах определяются не только количественными изменениями в содержании наночастиц, но также связаны с возможными изменениями структуры материала (плотностью упаковки частиц в агрегатах, пространственным рапределением агрегатов, распределением частиц по размерам). В разбавленных системах, содержащих изолированные наночастицы, изменения их концентрации отражают только количественные изменения фотоактивных центров. Таким образом, исследование нелинейно-оптических свойств разбавлен- ных золей отражает именно чистое количественное влияние КT CdS на измеряемые характеристики. КT $\mathrm{CdS}$ проявляют как отрицательные, так и положительные рефрактивные нелинейные свойства $[2,3,5,6]$. В литературе исследования КT CdS на длине волны 532 нм при наносекундной длительности представлены главным образом для полимерных пленок $[2,8,11-13]$, при этом проявляются противоположные нелинейные рефрактивные свойства. Как показано в работах $[3,14]$, знак нелинейной рефракции зависит от соотношения диаметра квантовых точек и воздействующей частоты излучения, а также от интенсивности падающего света. Если интенсивность превышает величину, при которой начинает доминировать поглощение свободными носителями, знак рефракции может измениться. Целью настоящей работы является исследование жидких золей с низкой концентрацией $\mathrm{CdS}$ при наносекундном воздействии, что позволит внести последовательность в изучение и дополнить понимание нелинейных характеристик $\mathrm{CdS}$ и природы взаимодействия квантовых точек с матрицей.

\section{2. Техника и методы эксперимента}

В качестве исходных компонентов для синтеза квантовых точек в настоящей работе использовались водные растворы $\mathrm{Cd}\left(\mathrm{NO}_{3}\right)_{2}$ и $\mathrm{Zn}\left(\mathrm{NO}_{3}\right)_{2}$, а также водный раствор 


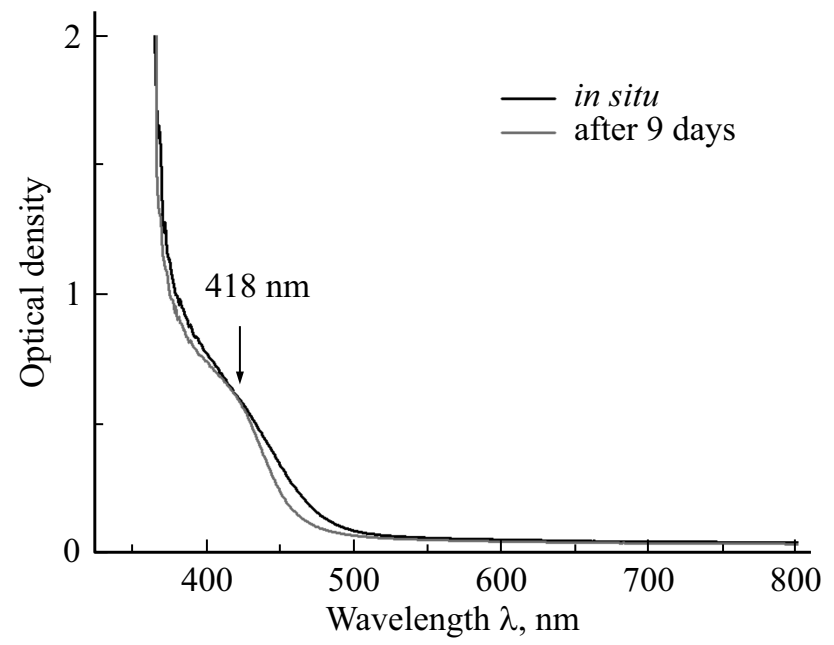

Рис. 1. Спектры поглощения золя $(8.4 \mathrm{MM})$ in situ и через 9 дней. Основной пик поглощения $(\lambda=418$ нм $)$ становится более выраженным из-за преобладания наночастиц одного диаметра.

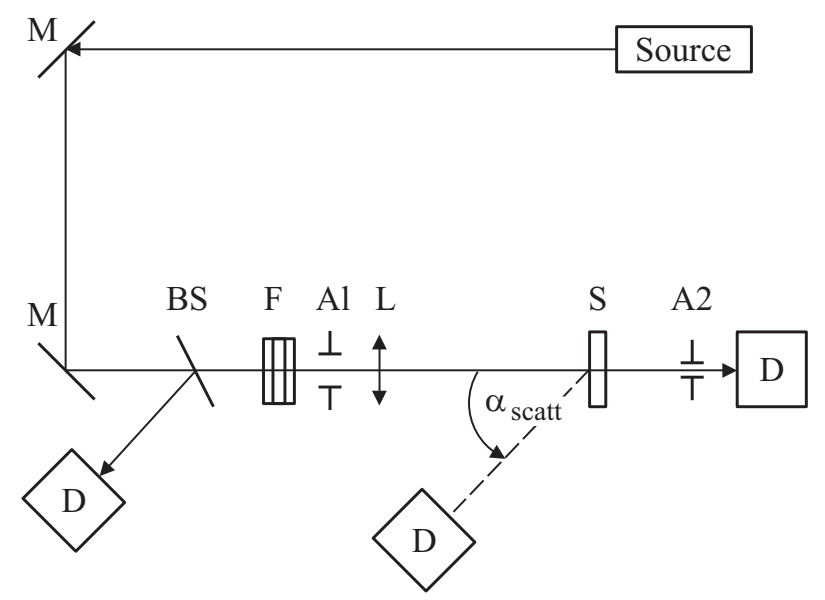

Рис. 2. Оптическая схема эксперимента по $z$-сканированию, с возможностью регистрации рассеянного сигнала. Source YAG:Nd-лазер, M - зеркала, BS - светоделительная пластина, F - нейтральные фильтры, А1 и А2 - апертуры, $\mathrm{L}$ - положительная линза с фокусным расстоянием $15 \mathrm{~cm}$, $\mathrm{S}$ - образец, D - приемники излучения, $\alpha_{\text {scatt }}-$ угол рассеяния.

$\mathrm{Na}_{2} \mathrm{~S}$. Для стабилизации наночастиц сульфидов металлов в реакционную смесь добавлялись растворы высокомолекулярного PVP (молярная масса $M=1300000$ г/моль) в пропаноле-2. Жидкая фаза золя, полученного при смешивании исходных растворов, представляла собой смесь пропанола-2 и воды. Высокомолекулярный поливинилпирролидон (HMW-PVP) был выбран согласно предварительно проведенным исследованиям в качестве высокостабильной и гомогенной матрицы, обеспечивающей стабилизацию формирующихся квантовых точек.

Спектры поглощения регистрировались с помощью спектрофотометра Lambda 1050 (Perkin Elmer). Спектры линейного поглощения оставались стабильными в течение длительного времени (см. рис. 1). Однако сравнение результатов измерений для растворов $\mathrm{CdS} / \mathrm{ZnS}-\mathrm{PVP}$ in situ и через 9 дней показало, что со временем нелинейные характеристики золей ухудшались. Коэффициенты двухфотонного поглощения $\beta$ (см/Вт) свежеприготовленного и девятидневного растворов различались вдвое, нелинейные рефрактивные свойства были потеряны практически полностью. Для исследования нелинейных характеристик синтезированных золей использовалось излучение второй гармоники YAG: $\mathrm{Nd}^{3+}$-лазера с длиной волны $\lambda_{\mathrm{ex}}=532 \mathrm{нм}$, длительностью импульса $\tau_{p}=5 \mathrm{Hc}$, энергией в импульсе $E_{p}=3-125$ мкДж. Оптическая схема эксперимента по z-сканированию приведена на рис. 2.

Гауссов пучок собирался линзой с фокусным расстоянием $F=15$ см. Образец (кювета с золем) перемещался вдоль главной оптической оси, проходя последовательно положения до фокусной плоскости (соответствовали отрицательным $z$ ) и после (соответствовали положительным $z$ ). Энергия на выходе из кюветы измерялась фотометром (OPHIR, PE9) в двух режимах, „с закрытой апертурой“ и „с открытой апертурой“, что позволило отделить вклад нелинейной рефракции от нелинейного поглощения и рассеяния. Для оценки вклада нелинейного рассеяния использовались показания третьего приемника (OPHIR, PD10), расположенного под углом к передней стенке кюветы с образцом. Толщина образца была выбрана с учетом критерия Рэлея и составляла 2 мм.

\section{3. Обсуждение результатов}

На рис. 3 приведен спектр поглощения одного из четырех приготовленных растворов, различающихся массовым содержанием $\mathrm{CdS}$ (табл. 1) Полученная форма спектра характерна для наночастиц $\mathrm{CdS}[15,16]$, отчетливо видна полоса основного экситонного перехода при

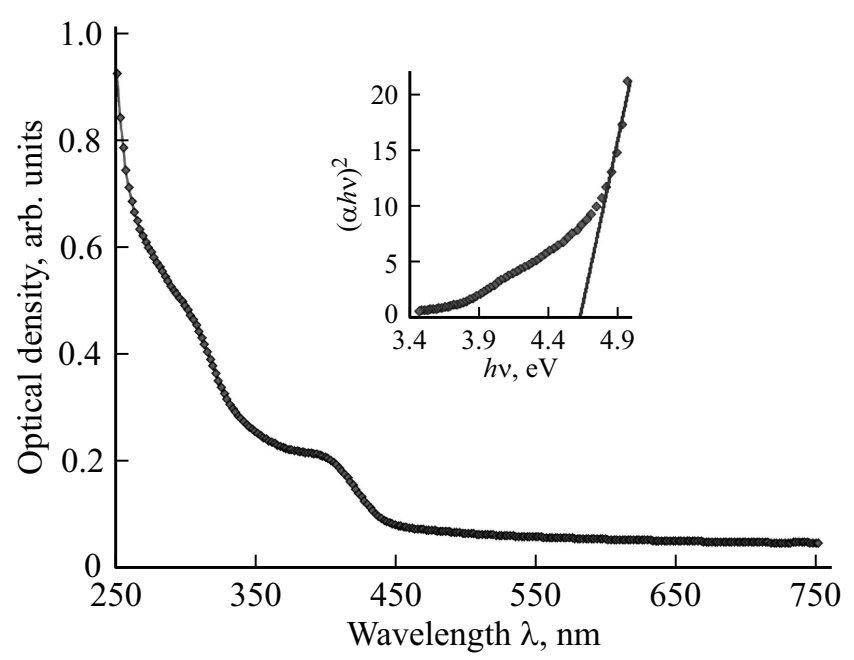

Рис. 3. Спектр поглощения синтезированного золя $\mathrm{CdS} / \mathrm{ZnS}-\mathrm{HMW}-\mathrm{PVP}$ с концентрацией $\mathrm{CdS} C=2.9 \mathrm{MM}$. На вставке - аппроксимация зависимостью $(\alpha h v)^{2}=f(h v)$. 
Таблица 1. Соотношение химических компонентов при приготовлении золей $\mathrm{CdS} / \mathrm{ZnS}$

\begin{tabular}{c|c|c|c|c|c|c}
\hline \multirow{2}{*}{$\begin{array}{c}\text { Концентрация CdS, } \\
\text { моль/л }\end{array}$} & \multicolumn{5}{|c}{ Массовая доля исходного компонента, \% } \\
\cline { 2 - 7 } & $\mathrm{H}_{2} \mathrm{O}$ & Propanol-2 & $\mathrm{Cd}\left(\mathrm{NO}_{3}\right)_{2} \cdot 4 \mathrm{H}_{2} \mathrm{O}$ & $\mathrm{Zn}\left(\mathrm{NO}_{3}\right)_{2} \cdot 6 \mathrm{H}_{2} \mathrm{O}$ & $\mathrm{Na}_{2} \mathrm{~S} \cdot 9 \mathrm{H}_{2} \mathrm{O}$ & $\mathrm{PVP}$ \\
\hline $8.4 \cdot 10^{-4}$ & 33.44 & 64.09 & 0.33 & 0.49 & 0.02 & 1.63 \\
$1.4 \cdot 10^{-3}$ & 33.43 & 64.08 & 0.33 & 0.49 & 0.04 & 1.63 \\
$2.8 \cdot 10^{-3}$ & 33.41 & 64.06 & 0.33 & 0.49 & 0.08 & 1.63 \\
$4.2 \cdot 10^{-3}$ & 33.40 & 64.03 & 0.33 & 0.49 & 0.12 & 1.63
\end{tabular}

длинах волн $\lambda=400-430$ нм. Ширина полосы определяется распределением наночастиц по размерам [17]. Также видно, что в спектре разрешается полоса, соответствующая переходу $1 P(e)-1 P_{3 / 2}(h)$ в ультрафиолетовой области $(\lambda=290-300$ нм $)$, по энергии близкая к 4.1 эВ.

Ширина запрещенной зоны синтезированных нанокристаллов рассчитывалась по уравнению Таука [18], которое для прямозонных полупроводников выглядит следующим образом:

$$
(\alpha h v)^{2}=A\left(h v-E_{g}\right),
$$

где $h v$ - энергия фотона, $E_{g}$ - ширина запрещенной зоны полупроводника, $A-$ константа, $\alpha-$ коэффициент поглощения. Спектры, построенные в координатах $(\alpha h v)^{2}=f(h v)$, позволили определить $E_{g}$ путем линейной экстраполяции полученных кривых к оси энергий. На основании данных [19] по величине изменения ширины запрещенной $\Delta E_{g}=E_{g}-E_{g \text { bulk }}$, где $E_{g \text { bulk }}$ - ширина запрещенной зоны объемного $\mathrm{CdS}$, в рамках модели, предложенной в [20], была проведена оценка диаметра $D$ наночастицы $\mathrm{CdS}$ по формуле

$$
D=3.062 / \sqrt{\Delta E_{g}},
$$

в которую диаметр наночастицы $D$ входит в нм и величина $\Delta E_{g}$ в эВ. Полученные величины сведены в табл. 2.

Похожие значения $E_{g}$ были получены авторами в работе [21] для CdS-золей, синтезированных аналогичным методом, и превышали $E_{g}$ макроскопического $\mathrm{CdS}$ $\left(E_{g \text { bulk }} \approx 2.4\right.$ эВ при $\left.293 \mathrm{~K}[22]\right)$ вследствие квантоворазмерного эффекта. Столь же малые размеры частиц $2.0-2.1$ нм CdS-золей в присутствии PVP наблюдались ранее в $[23,24]$.

Таблица 2. Диаметры синтезированных квантовых точек и величины запрещенной зоны

\begin{tabular}{c|c|c|c}
\hline $\begin{array}{c}\text { Оптическая } \\
\text { плотность, } \\
\text { отн.ед. }\end{array}$ & $\begin{array}{c}\text { Концентрация } \\
\mathrm{CdS}, \text { мM }\end{array}$ & $\begin{array}{c}E_{g}, \\
\text { э }\end{array}$ & $\begin{array}{c}\text { Диаметр } \\
\text { CdS, нм }\end{array}$ \\
\hline 0.05 & 0.8 & 4.6 & 2.0 \\
0.06 & 1.4 & 4.5 & 2.0 \\
0.21 & 2.8 & 4.8 & 2.1 \\
0.30 & 4.2 & 4.8 & 2.1
\end{tabular}

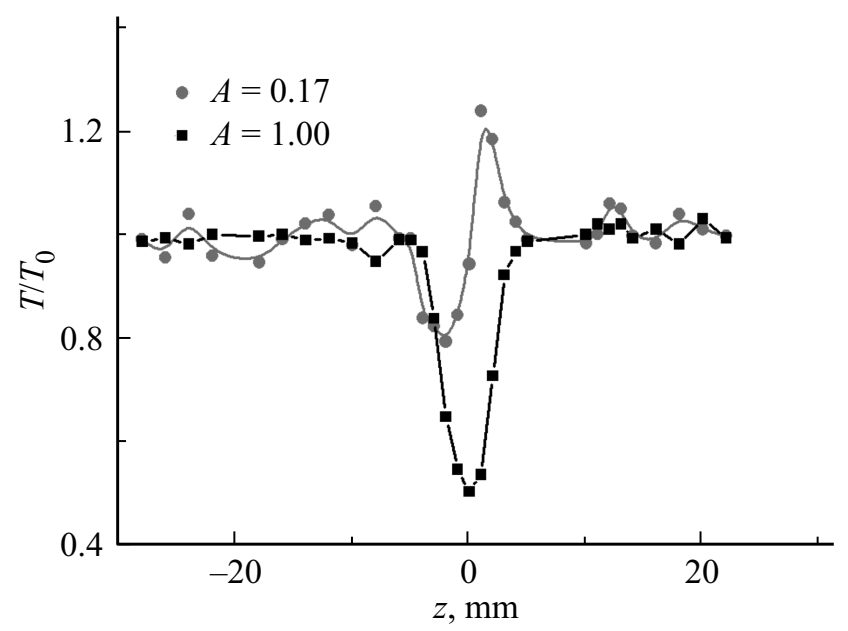

Рис. 4. Результаты $z$-сканирования золя $\mathrm{CdS} / \mathrm{ZnS}-\mathrm{HMW}-\mathrm{PVP}$ $(C=8.4 \mathrm{MM})$. Точки - экспериментальные значения нормированного пропускания в режиме открытой апертуры $(A=1)$ и в режиме закрытой апертуры $(A \approx 0.2)$ (с удалением вклада поглощения и рассеяния). Линии проведены для наглядности. Интенсивность излучения $I=0.4$ ГВТ/.м².

Длина волны лазерного возбуждения $\lambda_{\mathrm{ex}}=532$ нм соответствовала области прозрачности исследуемых золей (рис. 3), при высоких интенсивностях высока вероятность двухфотонного поглощения, соответствующего переходу 4.6-4.7 эВ. На рис. 4 показаны результаты измерения нормированного пропускания лазерного излучения, $T / T_{0}$, в зависимости от координаты $z$ для раствора с максимальным содержанием CdS. При исследованиях нелинейных характеристик синтезированных золей использовались концентрации, в 2 раза превышающие значения, приведенные в табл. 1. В режиме закрытой апертуры вблизи фокусной плоскости $(z=0)$ провал сменился пиком на кривой пропускания, что отвечает положительной нелинейной рефракции (самофокусировка излучения). Удаление апертуры показало вклады нелинейного рассеяния и поглощения. В случае открытой апертуры относительная погрешность составила $4.8 \%$, в случае закрытой $10 \%$. Основной вклад в разброс значений вносила пространственная нестабильность лазерного пучка, поэтому каждая точка на рис. 4 получена усреднением по 5 моноимпульсам. 


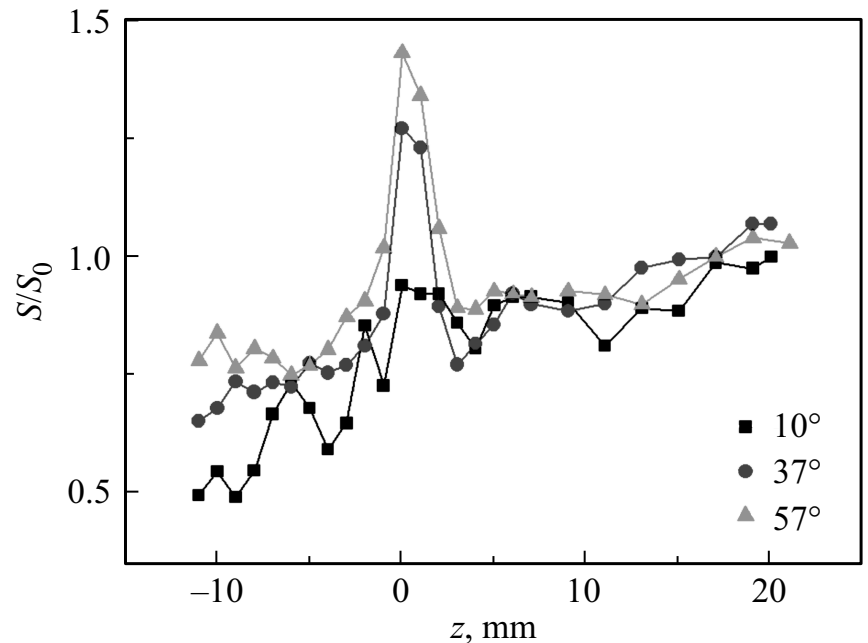

Рис. 5. Зависимость величины нормированного рассеяния $S / S_{0}$ от положения образца $z$ при разных углах регистрации (концентрация $C=8.4 \mathrm{MM}$ ).

Принимая во внимание малую величину входной энергии, преобладание нелинейной рефракции связанных носителей и положительный знак нелинейного показателя преломления, можно заключить, что энергия двухфотонного перехода приблизительно соответствует ширине запрещенной зоны исследуемых квантовых точек. Подобный случай описан в работах [3,14] при изучении механизмов нелинейного пропускания в наночастицах. По нашему мнению, не стоит исключать также и влияние молекулярного окружения. Например, в работах [4-6] показано, что при исследованиях КТ CdS под действием фемтосекундных лазерных импульсов на длинах волн 750-800 нм величина $\gamma(\gamma-$ нелинейный коэффициент рефракции связанных носителей, связан с нелинейным показателем преломления через соотношение $n_{2}=c n_{0} \gamma / 40 \pi[14]$, где $c-$ скорость света, $n_{0}-$ линейный показатель преломления) отклонялась как в сторону бо́льших, так и в сторону меньших значений в зависимости от матрицы окружения (см. обзорную таблицу в [6]).

Вклад нелинейного рассеяния в снижение пропускания регистрировался при четырех разных углах с

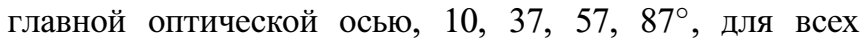
четырех концентраций растворов. Из рис. 5 видно, что величина нормированного рассеяния $S / S_{0}$ (где $S_{0}-$ энергия рассеянного излучения в точке $z=20 \mathrm{Mм}$ ) изменяется от 0.5 до 1 по мере движения образца во всем диапазоне измерений в ходе каждой серии эксперимента, из чего следует увеличение линейного рассеяния. Рост линейного рассеяния связан с незначительным увеличением температуры раствора, так как при наносекундных длительностях импульса квантовые точки отдают тепло окружающей среде. Линейное рассеяние в коллоидных растворах квантовых точек может быть вызвано присутствием их агрегатов. Однако низкое линейное поглощение в исследуемом диапазоне и отсутствие зависимости амплитуды линейного рассеяния от концентрации (здесь не показано) дополнительно подтверждают однородность и дисперсность синтезированных золей. На рис. 5 обращает на себя внимание амплитуда рассеяния в фокусе $(z=0)$, которая превышает соседние значения в среднем в 1.5 раза (такая картина наблюдалась для всех образцов), что указывает на наличие нелинейного рассеяния. При этом обнаружено отличие угловых зависимостей рассеяния: линейное рассеяние уменьшается в направлении от главной оптической оси к $90^{\circ}$ (имеет коническую природу), в то время как величина нелинейного растет до $60^{\circ}$, возвращаясь к начальным значениям по мере приближения к $90^{\circ}$. Нелинейное рассеяние может быть обусловлено формированием оптических неоднородностей, возникающих вокруг фотоактивных центров при воздействии интенсивного лазерного излучения. Обнаружено, что с увеличением концентрации квантовых точек $\mathrm{CdS} / \mathrm{ZnS}$ в нашем случае пропорционально ей растет амплитуда нелинейного рассеяния (не показано). Для подтверждения тепловой природы рассеяния и сопоставления его вклада с двухфотонным поглощением (ДФП) проведены исследования при разной частоте следования лазерных импульсов (рис. 6). Обнаружено, что величина нормированного пропускания снижалась до 0.87 в фокальной плоскости при частоте 0.1 Гц, что соответствовало вкладу чистого двухфотонного поглощения. При переходе к частоте 1 Гц провал сокращался до значения 0.80, показывая суммарный вклад двух механизмов и доказывая, что природа термически индуцированного рассеяния носит кумулятивный характер. Таким образом, отношение вклада ДФП к нелинейному рассеянию составило $65: 35 \%$.

Так как толщина образца не превышала дифракционный критерий Рэлея (4.5 мм для нашей схемы), становится возможным использование уравнений в приближении

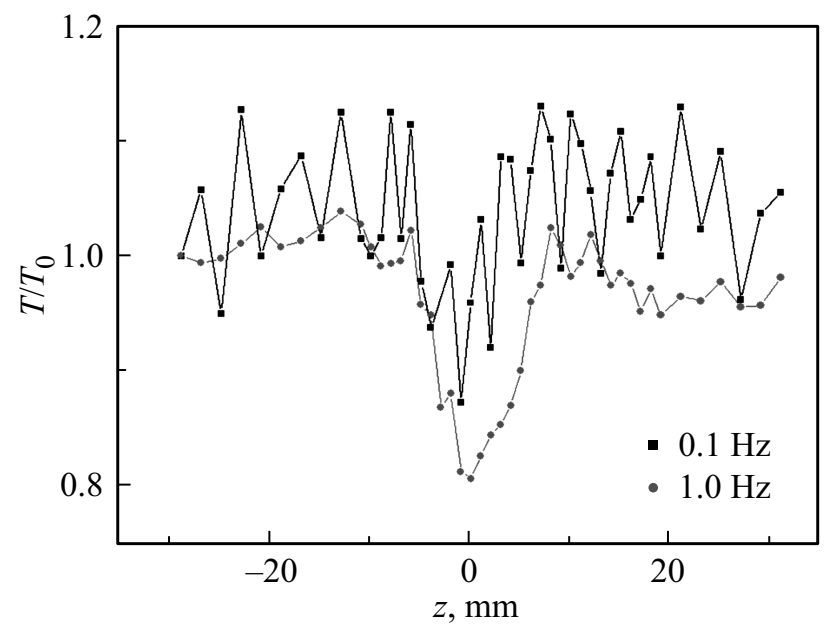

Рис. 6. Зависимость нормированного пропускания $T / T_{0}$ при разной скважности импульсов в режиме открытой апертуры для золя с концентрацией 8.4 мМ. 
Таблица 3. Нелинейные коэффициенты КТ $\mathrm{CdS} / \mathrm{ZnS}$, стабилизированных HMW-PVP $\left(I=0.4 \Gamma \mathrm{BT} / \mathrm{cm}^{2}\right)$

\begin{tabular}{c|c|c|c}
\hline $\begin{array}{c}\text { Концентрация } \\
\mathrm{CdS}, \mathrm{MM}\end{array}$ & $\begin{array}{c}T_{0}(532 \mathrm{Hм}), \\
\%\end{array}$ & $\begin{array}{c}\beta, \\
\mathrm{cm} / \Gamma \mathrm{BT}\end{array}$ & $\begin{array}{c}\gamma, \\
\mathrm{cm}^{2} / \mathrm{BT}\end{array}$ \\
\hline 1.7 & 88.2 & 1.9 & - \\
2.8 & 86.7 & 2.9 & $3 \cdot 10^{-14}$ \\
5.8 & 83.0 & 4.2 & - \\
8.4 & 82.8 & 5.3 & $7 \cdot 10^{-14}$
\end{tabular}

плоских волн. Воспользуемся выражениями для изменения фазы волны $\Delta \varphi$ и ее интенсивности $I$, приведенными в [14]:

$$
\left\{\begin{array}{l}
\frac{d \Delta \varphi}{d x}=k \Delta n, \\
\frac{d I}{d x}=-\left(\alpha_{0}+\beta I\right) I,
\end{array}\right.
$$

где $\Delta n=\gamma I+\sigma_{r} N-$ изменение показателя преломления, $k$ - модуль волнового вектора квазимонохроматического излучения, $\alpha_{0}$ - линейное поглощение, $x$ - глубина распространения излучения в образце, $\sigma_{r}$ - изменение показателя преломления при генерации свободных носителей плотности $N$. Коэффициенты двухфотонного поглощения $\beta$ (см/Вт) и нелинейной рефракции $\gamma\left(\mathrm{cm}^{2} / \mathrm{BT}\right)$ связаны с величинами мнимой и реальной частей нелинейной восприимчивости $\chi^{(3)}$ через соотношения [25]

$$
\begin{aligned}
& \chi_{\operatorname{Im}}^{(3)}=\frac{n_{0}^{2} \varepsilon_{0} c^{2}}{\omega} \beta, \\
& \chi_{\mathrm{Re}}^{(3)}=2 n_{0}^{2} \varepsilon_{0} c \gamma,
\end{aligned}
$$

где $\varepsilon_{0}$ - диэлектрическая проницаемость среды, $\omega-$ круговая частота воздействующего излучения. Рассчитанные коэффициенты нелинейной рефракции и двухфотонного поглощения с учетом нелинейного рассеяния для всех исследованных концентраций $\mathrm{CdS}$ представлены в табл. 3 .

Из табл. 3 видно, что для образца с максимальной концентрацией KT $\mathrm{CdS}$ коэффициент двухфотонного поглощения $\beta$ сравним с величиной, полученной для объемного материала, 5.4 см/ГВт [26]. Однако молярная концентрация объемного материала в $10^{4}$ раз выше (для объемного $\mathrm{CdS} \rho / M=33.4 \mathrm{M}, \rho-$ плотность), что свидетельствует об улучшении нелинейных характеристик наночастиц по сравнению с объемным полупроводником. Величина же $\gamma$ в несколько раз меньше приводимой в [26] величины для объемного $\mathrm{CdS}$, но может быть увеличена путем увеличения объемной доли квантовых точек уже в 10 раз относительно наибольшей из приведенных в табл. 3 при условии линейной зависимости $\gamma$ от концентрации. Используя формулы (4)-(5), найдем, что реальная часть нелинейной восприимчивости в 3 раза превышает мнимую.
Для определения величины интенсивности, при которой вкладом нелинейностей высших порядков еще можно пренебречь, была проведена серия экспериментов. Установлено, что золь, не содержащий серы (и, следовательно, квантовых точек), не проявлял нелинейных свойств во всем диапазоне интенсивностей. Следовательно, вклад буферного раствора в изменение нелинейного пропускания исключен.

Пороговой интенсивностью для возникновения двухфотонного поглощения является величина

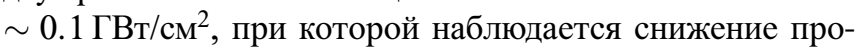
пускания исследуемого раствора в фокусной плоскости (рис. 7,a). При этом коэффициент ДФП оставался практически постоянным $(\beta=5.5 \pm 0.1 \mathrm{~cm} / Г В \mathrm{~B})$ в диапазоне интенсивностей до 0.4 ГВт/см ${ }^{2}$ (рис. 7,b). Уменьшение коэффициента $\beta$ при более высоких плотностях энергии связано с увеличением числа поглощающих свободных
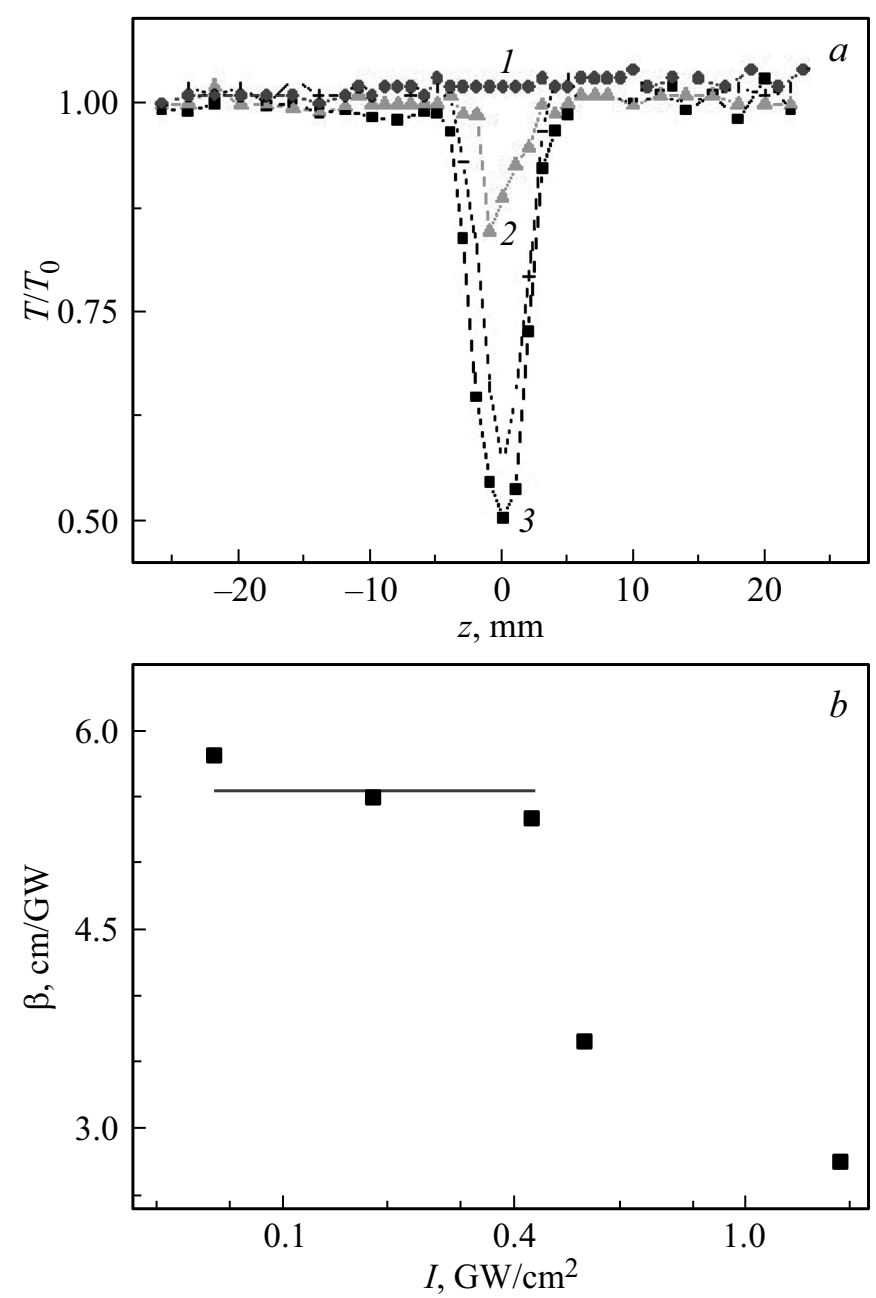

Рис. 7. $a-$ нормированное пропускание золя $\mathrm{CdS} / \mathrm{ZnS}-\mathrm{PVP}$ $(C=8.4 \mathrm{MM}) \quad$ в режиме открытой апертуры при различной пиковой интенсивности, ГВт/см ${ }^{2}: 1-(0.02-0.05)$, $2-(0.1-0.2), 3-(0.4-0.5) . \quad b-$ соответствующие расчетные значения $\beta$ для каждого диапазона интенсивностей (точки) и результат аппроксимации функцией $\beta=$ const для интенсивностей $<0.5$ ГВТ $/ \mathrm{cm}^{2}$ (прямая линия). 
носителей в результате ДФП и дополнительным вкладом нелинейного рассеяния в результате формирования термической линзы.

При малых концентрациях наночастиц взаимодействие электромагнитного поля с наночастицами может описываться теорией Лорентца-Ми [27]. Эффективное значение $\chi_{\text {eff }}^{(3)}$ может быть выражено как [28]

$$
\chi_{\mathrm{eff}}^{(3)}=(1-p) \chi_{m}^{(3)}+f^{4} p \chi_{s}^{(3)},
$$

где $\chi_{m}^{(3)}$ - диэлектрическая восприимчивость третьего порядка дисперсионной среды, $p$ - объемная доля наночастиц, $\chi_{s}^{(3)}$ - диэлектрическая восприимчивость третьего порядка наночастиц, $f$ - отношение интенсивности внешнего электромагнитного поля $E_{0}$ и поля внутри наночастицы $E_{s}, f=E_{s} / E_{0}$. Учитывая малую разницу в размерах наночастиц и низкую концентрацию в золях, можно предположить, что значения $f$ и $\chi_{s}^{(3)}$ во всех исследованных золях близки. Поэтому различие в значениях $\chi_{\mathrm{eff}}^{(3)}$ будет определяться объемной долей наночастиц $p$.

На рис. 8 сведены результаты исследований концентрационных зависимостей коэффициентов двухфотонного поглощения для квантовых точек разных диаметров: 2 нм (сплошная прямая) и 4 нм (штриховая), взятые из работы [1]. Видно, что для квантовых точек одного диаметра зависимость $\beta$ от концентрации КT $\mathrm{CdS}$ линейная, что закономерно по причине увеличения числа поглощающих центров. Известно, что для квантовых точек большего диаметра величины $\beta$ больше по причине увеличения нелинейной восприимчивости $\chi^{(3)}$ [29] и при условии, что диаметр квантовых точек меньше боровского радиуса экситона (для КТ $\mathrm{CdS} a_{\mathrm{B}}=30 \AA$ ). Однако из рис. 8 виден различный наклон аппроксимационных прямых и, следовательно, разная величина сечения двухфотонного поглощения $\sigma_{\mathrm{TPA}}\left(\beta \propto \sigma_{\mathrm{TPA}} C\right.$,

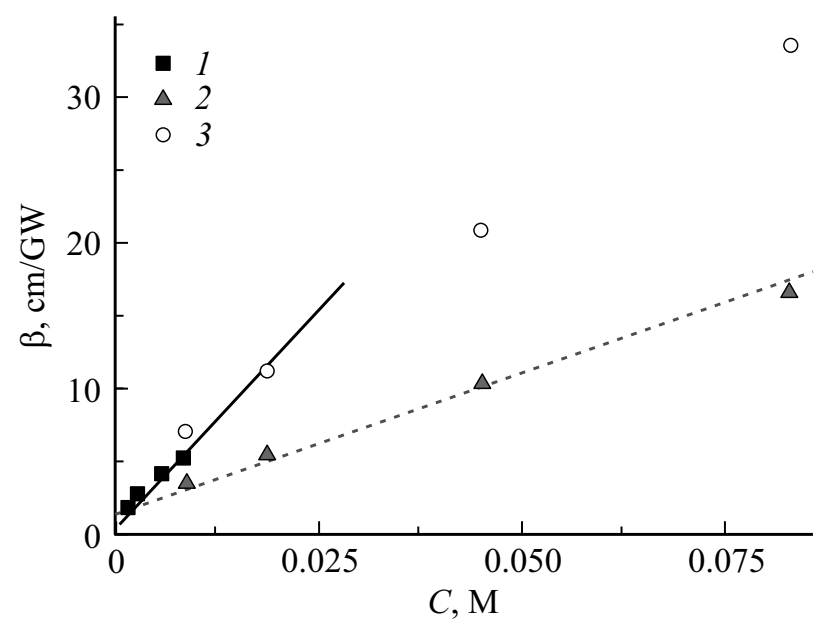

Pис. 8. Зависимости коэффициентов ДФП $\beta$ от концентрации КТ $\mathrm{CdS}$ в золях: 1 - данные из табл. 3,2 - данные [1] с вычетом нелинейности растворителя, 3 - возможные $\beta$, полученные умножением данных [1] на 2.

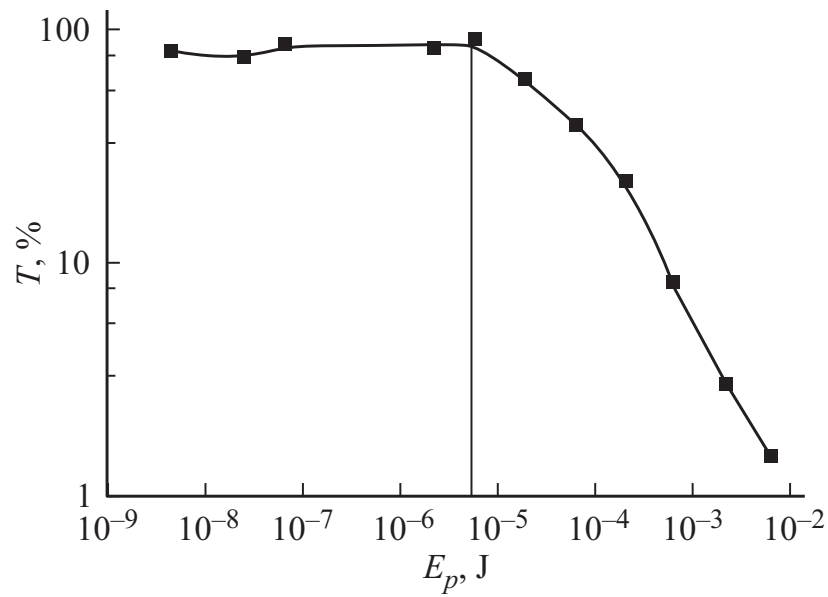

Рис. 9. Нелинейное пропускание золя $\mathrm{CdS} / \mathrm{ZnS}-\mathrm{HMW}-\mathrm{PVP}$

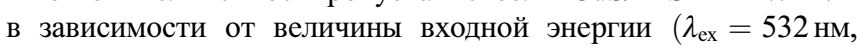
$\left.\tau_{p}=12 \mathrm{Hc}\right)$.

где $C$ - концентрация КТ [30]). В нашем случае величина $\sigma_{\text {ТРА }}$ составила $5.5 \cdot 10^{-46} \mathrm{~cm}^{4} \cdot \mathrm{c} /$ фотон. Сравнивая значения $\beta$, полученные при интенсивности 1 ГВт/ $\mathrm{cm}^{2}$, приведенные в работе [1], с нашими результатами (рис. 7, $b$ ), при прочих равных условиях, заметим, что в этом диапазоне интенсивностей необходимо учитывать влияние нелинейностей высоких порядков $\left(\chi^{(5)}\right.$ и др.), что не отражено в [1]. Из рис. $7, b$ следует, что при $I=1 \Gamma$ ГТ $/ \mathrm{cm}^{2}$ коэффициенты ДФП в 2 раза меньше, чем в случае $\chi^{(3)}$-приближения, поэтому, если мы умножим на 2 значения $\beta$ штриховой прямой 2 на рис. 8 , то получим единую концентрационную зависимость для квантовых точек разных диаметров, что подтвердит независимость сечения двухфотонного поглощения от содержания КТ в золе.

Таким образом, разница в наклонах прямых (рис. 8) может быть обусловлена следующими причинами: различием диаметров КТ $\mathrm{CdS}$ (или в величинах $\chi^{(3)}$ ), влиянием нелинейностей высоких порядков.

Синтезированные золи были исследованы в оптической схеме по оптическому ограничению (см., например, [31]). Кювета (2 мм) помещалась в фокус телескопической системы с двумя положительными линзами (фокусное расстояние 50 мм). На выходе из телескопа излучение собиралось длиннофокусной линзой в телесный угол 1.5 мрад и регистрировалось приемником PD10 (OPHIR). Величина входной энергии варьировалась с помощью нейтральных светофильтров. Согласно результатам $z$-сканирования, двухфотонное поглощение проявляется при интенсивности $0.1-0.2$ ГВт/см ${ }^{2}$ (рис. 7, $a$, кривая 2), на кривой ограничения (см. рис. 9) это соответствует энергии $\sim 5 \cdot 10^{-6}$ Дж. Снижение пропускания на 20\% (так называемый порог ограничения) совпадало c началом эффективного двухфотонного поглощения, а дальнейшее снижение пропускания связано с сопутствующими процессами поглощения на свободных носителях, нелинейной рефракции, нелинейного рассеяния 
на тепловых неоднородностях у поверхностей квантовых точек.

При нанесении синтезированных золей на твердые поверхности необходимо контролировать толщину получаемой пленки, которая должна составлять не менее 10 мкм для обеспечения высокой концентрации квантовых точек и устойчивости к лазерному воздействию.

\section{4. Заключение}

Показано, что синтезированные золи, содержащие полупроводниковые квантовые точки $\mathrm{CdS} / \mathrm{ZnS}$ диаметром 2 нм, стабилизированные высокомолекулярным поливинилпирролидоном, обладают высокой стабильностью и однородностью на протяжении длительного времени. Результаты исследований золей $\mathrm{CdS} / \mathrm{ZnS}-\mathrm{PVP}$ in situ под действием импульсов интенсивного лазерного излучения с параметрами 532 нм, 5 нс показали, что механизмами снижения нелинейного пропускания являются двухфотонное поглощение, положительная нелинейная рефракция и нелинейное рассеяние. Основная роль в снижении пропускания принадлежит нелинейной рефракции, вклады ДФП и нелинейного рассеяния составили около 20 и 10\% соответственно.

Получено подтверждение теплового характера нелинейного рассеяния (обусловленного формированием тепловых линз около поверхностей квантовых точек). Приведенные коэффициенты двухфотонного поглощения значительно превышают значения для объемного материала (при тех же условиях). Определен интервал действия нелинейностей третьего порядка по интенсивности: $I \leq 0.4$ ГВт/см ${ }^{2}$. Проведено сравнение концентрационных зависимостей коэффициентов ДФП для квантовых точек $\mathrm{CdS} / \mathrm{ZnS}$ разных диаметров, которое подтвердило их линейный характер. Уточнено значение сечения двухфотонного поглощения: $5.5 \cdot 10^{-46} \mathrm{~cm}^{4} \cdot \mathrm{c} /$ фотон. Полученные результаты могут быть использованы при расчетах и конструировании аттенюаторов лазерного излучения в видимом диапазоне, а также при создании новых полимерных покрытий; перспективным представляется и использование подобных материалов в схемах генерации многомерных лазерных солитонов..

Работа поддержана Российским научным фондом, грант 18-12-00075.

\section{Список литературы}

[1] N. Venkatram, R.S.S. Kumar, D.N. Rao. J. Appl. Phys., 100, 074309 (2006).

[2] C. Jing, X. Xu, X. Zhang, Z. Liu. J. Chu. J. Phys. D: Appl. Phys., 42, 075402 (2009).

[3] R.A. Ganeev, A.I. Ryasnyansky, R.I. Tugushev, T. Usmanov. J. Opt. A: Pure Appl. Opt., 5, 409 (2003).

[4] J. He, W. Ji, G.H. Ma, S.H. Tang, E.S.W. Kong, S.Y. Chow, X.H. Zhang, Z.L. Hua, J.L. Shi. J. Phys. Chem. B, 109, 4373 (2005).
[5] H.M. Gong, X.H. Wang, Y.M. Du, Q.Q. Wang. J. Chem. Phys., 125, 024707 (2006).

[6] J. Szeremeta, M. Nyk, D. Wawrzynczyk, M. Samoc. Nanoscale, 2013 (DOI: 10.1039/c3nr33860f).

[7] P. Ghosh, E. Ramya, P.K. Mohapatra, D. Kushavah, D.N. Rao, P. Vasa, K.C. Rustagi, B.P. Singh. Mater. Sci., 2016 (https://arxiv.org/abs/1606.02088).

[8] H. Du, G.Q. Xu, W.S. Chin, L. Huang, W. Ji. Chem. Mater., 14, 4473 (2002).

[9] Yu.P. Rakovich, M.V. Artemyev, A.G. Rolo, M.I. Vasilevskiy, M.J.M. Gomes. Phys. Status Solidi B, 224, 319 (2001).

[10] J. He, W. Ji, G.H. Ma, S.H. Tang, H.I. Elim, W.X. Sun, Z.H. Zhang, W.S. Chin. J. Appl. Phys., 95, 6381 (2004).

[11] R.E. Schwerzel, K.B. Spahr, J.P. Kurmer, V.E. Wood, J.A. Jenkins. J. Phys. Chem. A, 102, 5622 (1998).

[12] R.A. Al Aloosi, H.A. Jawad. Intern. J. Adv. Res. Education \& Technology (IJARET), 3, 146 (2016).

[13] J.V. Antony, J.J. Pillai, P. Kurian, N.V.P. Nampoori, G.E. Kochimoolayil. New J. Chem., 2017 (DOI: 10.1039/C6NJ03665A).

[14] A.A. Said, M. Sheik-Bahae, D.J. Hagan, T.H. Wei, J. Wang, J. Young, V.E.W. Stryland. J. Opt. Soc. Am. B, 9, 405 (1992).

[15] S. Vempati, Y. Ertas, T. Uyar. J. Phys. Chem. C, 117, 21609 (2013).

[16] J.I. Kim, J. Kim, J. Lee, D.-R. Jung, H. Kim, H. Choi, S. Lee, S. Byun, S. Kang, B. Park. Nanoscale Res. Lett., 7, 482 (2012).

[17] M. Pattabi, B.S. Amma, K. Manzoor. Mater. Res. Bull., 42, 828 (2007).

[18] J. Tauc. Mater. Res. Bull., 3, 37 (1968).

[19] Н.С. Кожевникова, А.С. Ворох, А.А. Урицкая. Успехи химии, 84, 225 (2015).

[20] A1.L. Efros, A.L. Efros. Sov. Phys. Semicond., 16, 772 (1982).

[21] M.R. Kim, Y.-M. Kang, D.-J. Jang. J. Phys. Chem. C, 111, 18507 (2007).

[22] O. Zelaya-Angel, J.J. Alvarado-Gil, R. Lozada-Morales, H. Vargas, A. Ferreira da Silva. Appl. Phys. Lett., 64, 281 (1994).

[23] К.С. Евстропьев, Ю.А. Гатчин, С.К. Евстропьев, К.В. Дукельский, И.М. Кисляков, Н.А. Пегасова, И.В. Багров. Опт. и спектр., 120, 434 (2016).

[24] L. Sarayanan, S. Diwakar, R. Mohankumar, A. Pandurangan, R. Jayavel. Nanomater. Nanotechnol., 1, 42 (2001).

[25] J. Wei. Nonlinear Super-Resolution Nano-Optics and Applications [Springer Ser. in Optical Sciences, 191, 25 (2015)].

[26] H.P. Li, C.H. Kam, Y.L. Lam, W. Ji. Optics Commun., 190, 351 (2001).

[27] M. Kerker. The Scattering of Light and Other Electromagnetic Radiation (N. Y., Academic Press, 1969).

[28] T. Ning, P. Gao, W. Wang, H. Lu, W. Fu, Y. Zhou, D. Zhang, X. Bai, E. Wang, G. Yang. Physica E, 41, 715 (2009).

[29] Y. Masumoto, T. Takagahara. Semiconductor Quantum Dots Physics, Spectroscopy and Applications (N.Y., SpringerVerlag Berlin Heidelberg, 2002).

[30] R.W. Boyd. Nonlinear optics (N. Y., Academic, 2003).

[31] А.С. Панфутова. Автореф. канд. дис. (Санкт-Петербург, АО „ГОИ им. С.И. Вавилова“, 2016).

Редактор Л.В. Шаронова 


\section{Nonlinear optical properties of deluted CdS/ZnS sols stabilized by HMW-PVP}

A.S. Kulagina ${ }^{1,2}$, S.K. Evstropiev ${ }^{2,3}$, N.N. Rosanov ${ }^{2,3,4}$, V.V. Vlasov ${ }^{2}$

${ }^{1}$ Saint Petersburg Academic University, 194021 St. Petersburg, Russia

${ }^{2}$ Saint Petersburg National Research University of Information Technologies, Mechanics and Optics, 197101 St. Petersburg, Russia

${ }^{3}$ Vavilov State Optical Institute, 199053 St. Petersburg, Russia

${ }^{4}$ loffe Institute, 194021 St. Petersburg, Russia

Abstract Hybrid composites of $\mathrm{CdS} / \mathrm{ZnS}$ core-shell nanoparticles of $2 \mathrm{~nm}$ size embedded in high molecular weight polyvinylpyrrolidone (HMV-PVP) matrixes have been prepared and characterized. $\mathrm{CdS} / \mathrm{ZnS}$ semiconductor quantum dots smaller than the Bohr radius of the exciton possessing quantum-size effect are of interest for a wide range of nanophotonics applications. Nonlinear optical properties of diluted $\mathrm{CdS} / \mathrm{ZnS}$ sols were studied at $532 \mathrm{~nm}$ and $5 \mathrm{~ns}$ laser pulses by using the $z$-scan technique. Dependence of the nonlinear-optical coefficients on the CdS concentration has been obtained. We presented also nonlinear two-photon absorption (TPA) coefficient dependence on intensity, what made possible to find the contribution of free carriers to nonlinear absorption and define the boundary of high nonlinearities actions. Discussed are different mechanisms of optical limiting by prepared sols: two photon absorption, nonlinear scattering and nonlinear refraction. 